\title{
Harmonic Suppressed Slot Antennas Using Rectangular/Circular Defected Ground Structures
}

\author{
Mohammad Saeid Ghaffarian, Gholamreza Moradi, and Reza Zaker \\ Wave Propagation and Microwave Measurement Research Laboratory, Department of Electrical Engineering, \\ Amirkabir University of Technology, Tehran 15914, Iran \\ Correspondence should be addressed to Mohammad Saeid Ghaffarian, msghaffarian@aut.ac.ir
}

Received 19 October 2011; Accepted 20 May 2012

Academic Editor: Dalia N. Elshiekh

Copyright ( 12012 Mohammad Saeid Ghaffarian et al. This is an open access article distributed under the Creative Commons Attribution License, which permits unrestricted use, distribution, and reproduction in any medium, provided the original work is properly cited.

Two wide rectangle-shaped microstrip-fed 2.6-GHz slot antennas using defected ground structures (DGSs) with a low design complexity are proposed to achieve wideband harmonic suppression. To accomplish this, two rectangular DGSs (RDGSs) in the first antenna and two circular DGSs (CDGSs) in the second one with various dimensions are etched into the ground plane, which could have a wideband-stop characteristic. Simulated and measured reflection coefficients indicate that the two proposed structures effectively suppress the second and third harmonics up to $23 \mathrm{~dB}$ between 3.5 and $10.5 \mathrm{GHz}$ with a maximum ripple of $2.4 \mathrm{~dB}$. In addition, the radiation patterns and peak gains of the antennas can be suppressed at least $17 \mathrm{~dB}$ and $7.1 \mathrm{dBi}$, respectively, at the third harmonic frequency of $7.86 \mathrm{GHz}$.

\section{Introduction}

Microstrip antennas can be conventionally integrated with active RF devices, especially amplifiers. In these cases, to maximize power added efficiency (PAE) of the power amplifiers (PAs), several methods are carried out on the PA itself. Since these approaches caused RF front-end systems to have large size, complex structures, and difficulty to design, the active integrated antennas (AIAs) were proposed. AIAs are very widespread, but they suffer from undesired harmonic radiation which must be suppressed. To this purpose, in the conventional harmonic tuning circuits, additional components such as filters were employed which increase the size and cost of RF front-ends and yield an additional insertion loss $[1,2]$.

In the last decades, several methods have been researched in practice to decrease insertion loss and spurious radiation in slot and patch antennas with microstrip and CPW-fed method [3-8]. In [2], Itoh et al. have introduced a photonic bandgap structure (PBG) based on the periodic structure to tune the second harmonic in an active slot antenna. After that, there has been an increasing interest in applying PBG and electromagnetic bandgap (EBG) structures as well as defected ground structure (DGS). Afterwards, new structures using L-C resonators as spurline conductors or other structures which act like filters that positioned on a radiator or feeding line have been proposed [4-7]. Also, harmonic suppression is done by defecting rectangular slot on tubing stub in CPW slot antenna [8]. It should be noted that the designs of mentioned periodic structures are not simple because of having several design parameters, difficulties of the modeling, and complex pattern and therefore, their fabricating is difficult.

In this paper, two harmonic suppressed slot antennas (HSAs) with rectangular and circular DGSs (i.e., RDGS \& CDGS) are parametrically investigated. The antennas having low design complexity such as less-design parameters and simpler DGS patterns can be suitable options for broadband unwanted harmonic suppression. In addition, in order to get a better understanding of the suppression performance of the antennas, the current distribution and circuit model are also discussed.

\section{Antenna Design and Configuration}

Figure 1 shows the geometry of the two slot harmonic suppression antennas in which the first one (Figure 1(a)) consists of two unequal RDGS with parameters of WS1, LS1, 


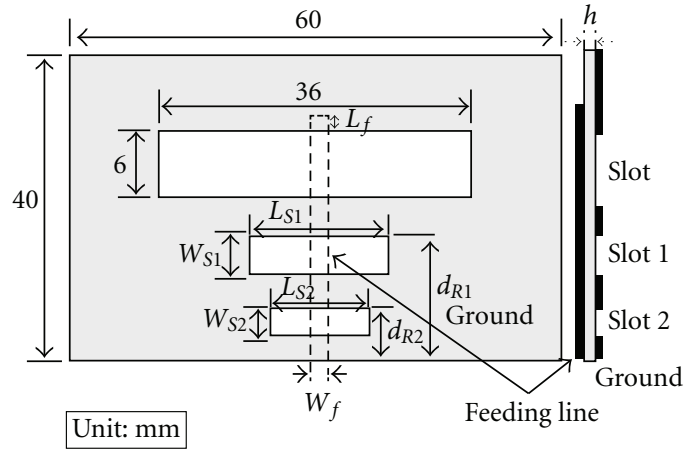

(a)

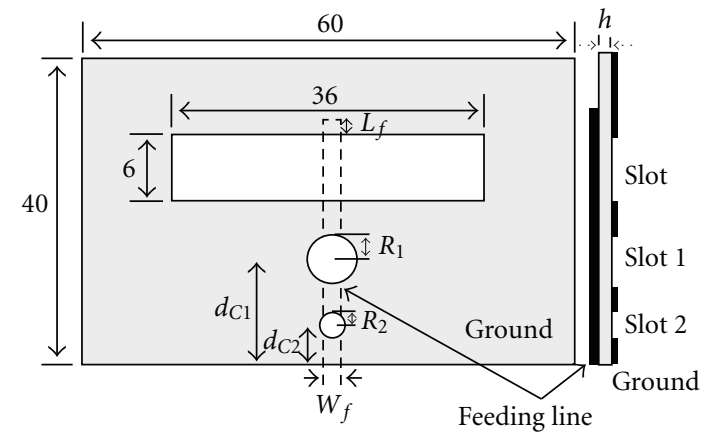

(b)

\begin{tabular}{|c|c|c|c|c|c|c|c|c|c|c|c|c|c|}
\hline Parameters & $W_{S 1}$ & $L_{S 1}$ & $W_{S 2}$ & $L_{S 2}$ & $R_{1}$ & $R_{2}$ & $W_{f}$ & $h$ & $L_{f}$ & $d_{R 1}$ & $d_{R 2}$ & $d_{C 1}$ & $d_{C 2}$ \\
\hline Values $(\mathrm{mm})$ & 3.5 & 11 & 2.5 & 6 & 3.8 & 1 & 1.1 & 0.508 & 1 & 14 & 8 & 11 & 5 \\
\hline
\end{tabular}

FIGURE 1: Configuration of the two proposed antennas with their optimal parameters: (a) geometry of rectangular DGS slot antenna and (b) geometry of circular DGS slot antenna.

dR1 and WS2, LS2, and dR2, and the other one (Figure 1(b)) has two unequal CDGS with parameters of $\mathrm{R} 1, \mathrm{dC} 1$ and $\mathrm{R} 2, \mathrm{dC} 2$. The antennas are fabricated on the substrate RO4003C with $0.5-\mathrm{oz}$ copper, whole dimension of $40 \times$ $60 \mathrm{~mm}^{2}, 0.508 \mathrm{~mm}$ height, and dielectric constant of 3.38 . The width of the microstrip-fed line is $W_{f}=1.1 \mathrm{~mm}$ for a $50 \Omega$ input impedance. The optimal physical parameters of both proposed antennas are summarized in a table and illustrated in Figure 1. To eliminate unwanted radiation at harmonic frequencies, two DGS-type slots (RDGS and CDGS) are etched into the ground plane exactly below the microstrip line and near the main radiation slot with a dimension of $6 \times 36 \mathrm{~mm}^{2}$. The proposed unequal slots can be represented as sequential shunt $\mathrm{L}-\mathrm{C}$ resonators, which have totally wideband-stop operation over the third harmonic frequency.

In the next section, it will be shown from parametric studies that the positions and dimensions of the DGS slots are important factors to suitably suppress the third harmonic frequency and tune the matching at the main resonance and the notched band.

Some of the popular DGSs are shown in Figure 2. These structures have their own distinguish properties which can be used for harmonic suppression. The reflection coefficients of rectangular, circular, triangular, spiral, dumbbell, and $\mathrm{H}$ shape DGS is shown in Figure 3. To give a better understanding of the performance of DGSs and comparison between them, the position and etched square area of DGSs are identical. The etched square of DGS is critical factor for determining the depth of suppression. Therefore, this factor is parametrically investigated in the next section. As seen from Figure 3, a little frequency shifting at the fundamental frequency and depth of suppression at the third harmonic frequency are important issues which should be noted. Hence, every pair of DGSs can be suitable for harmonic suppression in slot antenna if they are placed at the appropriate position. Because of decreasing of the context, the general properties of the mentioned DGSs such as the

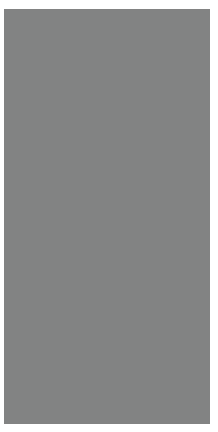

(a)

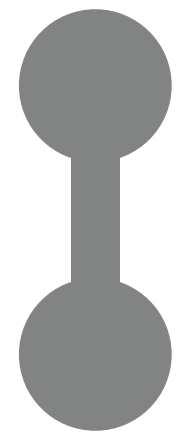

(d)

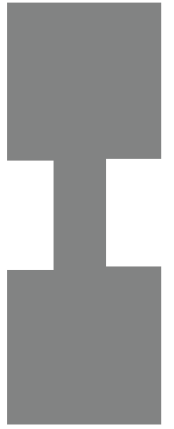

(b)

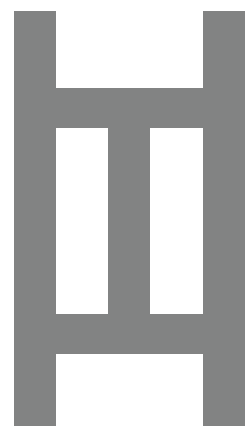

(e)

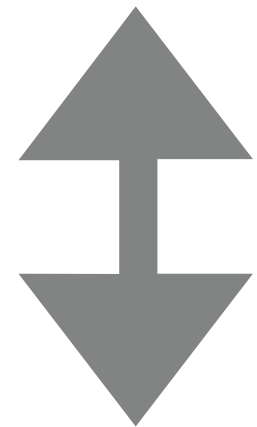

(c)

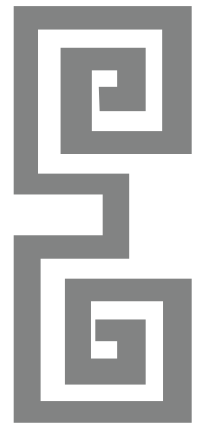

(f)
FIGURE 2: Different slots for DGS: (a) rectangular DGS, (b) square head DGS (dumbbell shape), (c) triangular DGS, (d) circular DGS, (e) H shape DGS, and (f) spiral DGS.

act of suppression and parametrically studies are examined for the rectangular and circular DGS's slot antenna, and their results can be ascribe to all of them.

In order to give a physical insight, the current distribution on the ground plane is investigated. Figure 4(a) shows the current in the absence of DGS filter, and Figures 4(b) and $4(\mathrm{c})$ demonstrate the disturbance in current distribution 


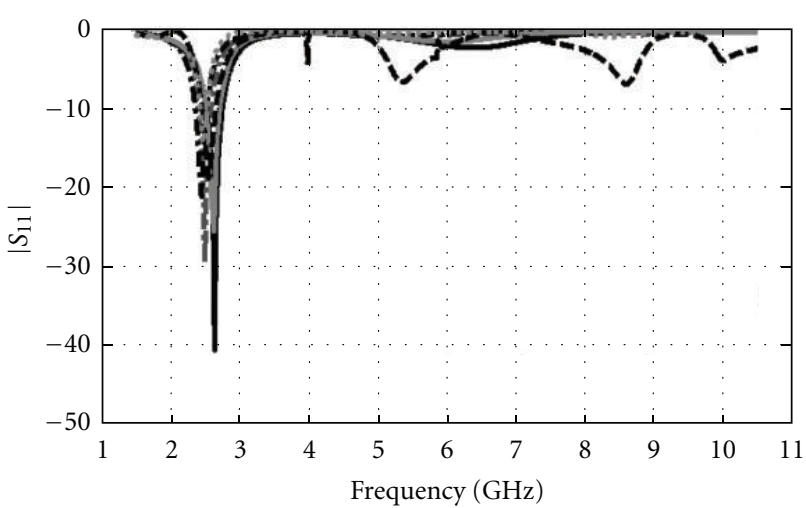

$$
\begin{aligned}
& \text {... Triangular shape - Rectangular shape } \\
& \text {..- Circular shape - - - Spiral shape } \\
& \text { - H shape ….. Dumbbell shape }
\end{aligned}
$$

FIgURE 3: Simulated reflection coefficients of HSA with different kinds of DGS. The position and etched square area of every DGS are identical.

which caused by DGS combinations. The electromagnetic field is mostly confined under the microstrip line. The return current on the ground plane is the negative image of the current distribution on the microstrip line. The return path of the current is fully disturbed using the DGS, and this current is confined to the perimeter of the perturbation and returns to the underneath of the microstrip line once the perturbation is over. By considering the maximum concentration of the return surface current on the ground plane, the length of the slot which contributes to inductance of the DGS is determined. The width of the slots is represented by the equivalent capacitances and the inductances and capacitances, are derived from the physical dimensions using quasistatic expressions for microstrip crosses, lines, and gaps which are presented in the open literatures [9]. The same approach is ascribed to CDGS slot antenna. On the basis of this observation, an equivalent circuit model is developed. Equivalent circuit model of RDGS slot antenna can be modeled based on the transmission line model. As shown in Figure 5, the proposed circuit model consists of two parts. The first part is the lossy model of two DGS slots that composed of two parallel RLC for each slot resonator and parallel capacitor that is due to relatively large fringing field at the step discontinuity plane on metallic ground surface [10]. The second part is the model of slot antenna that made of two short-circuited slot lines parallel (with the length of $L_{c s}=18 \mathrm{~mm}$, which is equal to the half-length $L_{s}$ of rectangular slot) with conductance $G_{s}$, representing the radiated power from the slot and parallel RC which is the admittance at the input of the open-ended microstrip stub which are obtained by equations that are mentioned in [11]. The optimized ADS-simulated results of the parameters of equivalent model are also shown in Figure 5. Figure 6 shows the reflection coefficients of the circuit model of RDGS antenna in comparison with the HFSS result. Since the parallel capacitance might cause changing in the impedance of the DGS section, it should be considered

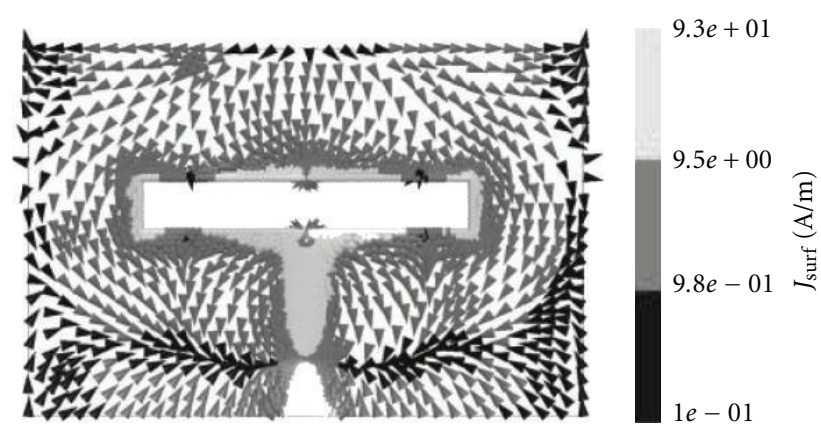

(a)

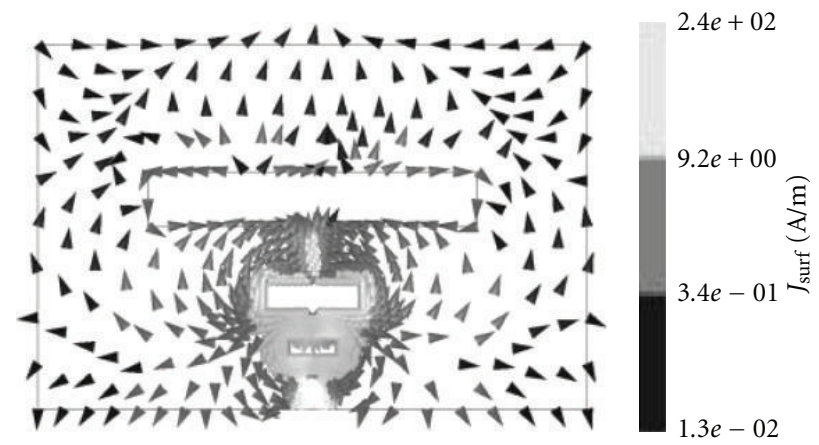

(b)

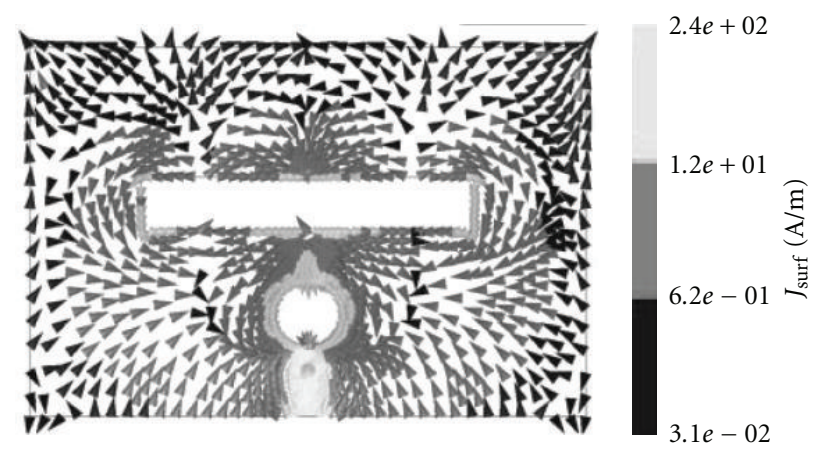

(c)

FIGURE 4: Simulated current distribution on the ground plane at the third harmonic frequency for (a) conventional slot antenna, (b) RDGS slot antenna, and (c) CDGS slot antenna.

for more precise modeling procedure. Circuit functionalities as filtering unwanted high-order harmonics can easily be accomplished by means of placing required DGS patterns without increasing circuit complexity. Each DGS provides its own distinctive characteristics depending on its geometries. It will be shown from parametric studies that the dimensions of the DGS slots are important factors to suitably suppress the third harmonic frequency.

\section{Simulated and Measured Results and Discussions}

The simulation is performed using high frequency structure simulator (HFSS). By using the DGS section, both the 


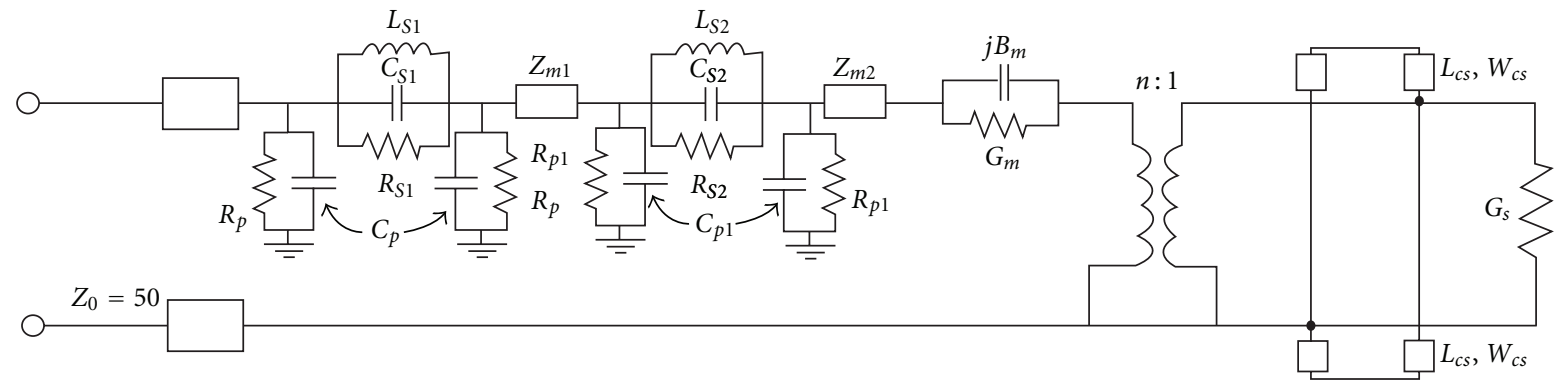

\begin{tabular}{|c|c|c|c|c|c|c|c|c|c|c|c|c|c|c|c|c|}
\hline Parameters & $R_{p}$ & $R_{p 1}$ & $C_{p}$ & $C_{p 1}$ & $L_{S 1}$ & $L_{S 2}$ & $C_{S 1}$ & $C_{S 2}$ & $R_{S 1}$ & $R_{S 2}$ & $n$ & $G_{s}$ & $L_{c s}$ & $W_{c s}$ & $Z_{m 1}$ & $Z_{m 2}$ \\
\hline Values & 0.11 & 1.43 & 4.05 & 62.6 & 94.5 & 43.6 & 64.95 & 119.5 & 5.72 & 4.72 & 2.28 & 0.12 & 18 & 1 & 75.8 & 71.1 \\
\hline
\end{tabular}

\begin{tabular}{|c|c|c|c|c|}
\hline Parameters & $R$ & $C$ & $L$ & Length \\
\hline Unit & $\mathrm{K} \Omega$ & $\mathrm{pF}$ & $\mathrm{nH}$ & $\mathrm{mm}$ \\
\hline
\end{tabular}

FIGURE 5: Circuit model for rectangular defected ground structure slot antenna.

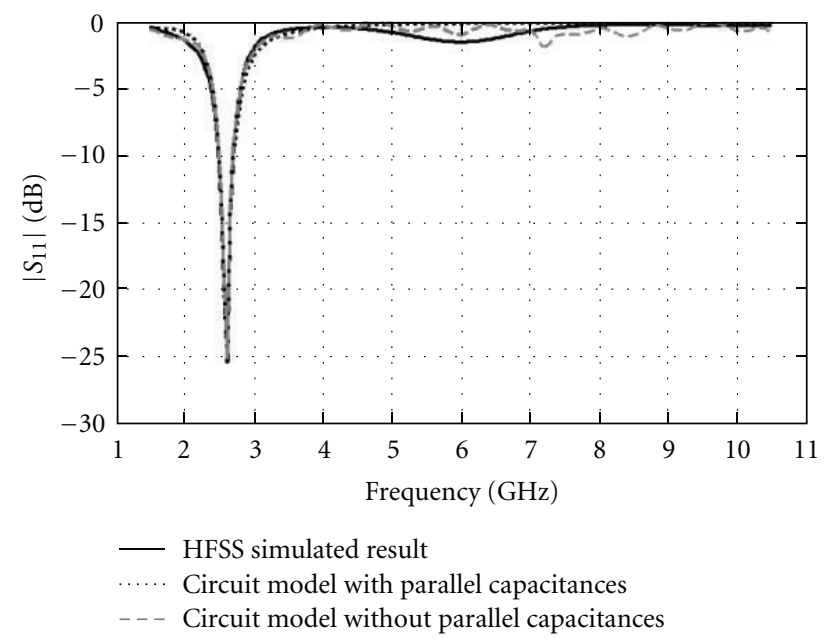

Figure 6: Reflection coefficients of equivalent circuit model of the harmonic suppression antenna.

effective permittivity and effective inductance of a microstrip line are increased [5]. The etched square area in the ground plane is critical factor for determining the depth of suppression. First, the parameters of the RDGS slot antenna are investigated. The change in the length in comparison with the change in the slots width is more effective for controlling the resonate frequency of RDGS slots. Figure 7(a) shows that when the length of DGS slots is altered, the depth of suppression is notably modified. The optimal ratio value of $L_{S 1} / L_{S 2}$ is 1.8. The same investigation is applied to CDGS slot antenna by changing the radius of slots in which the relevant parametric results are indicated in Figure 7(b). It is clearly seen that by decreasing the ratio of $R_{1} / R_{2}$, the quality of the suppression is extremely degraded especially between 5 and $9 \mathrm{GHz}$. It is interesting to point out that our studies show that the area of small DGS slots has important role in the value of input impedance at the third harmonic frequency. When the area of the slot is reduced, the input impedance is increased at the third harmonic frequency and makes that we have not reactively termination at this harmonic.

In order to validate the designs, the optimized structures of proposed HSAs were manufactured and measured carefully. Figures 8 and 9 show the simulated and measured reflection coefficients and the measured input impedances of the proposed rectangular and circular DGS-type slot antennas, respectively. To compare the quality of harmonic suppression, a conventional wide slot antenna without DGS is fabricated and measured. Figures $8(a)$ and $9(a)$ indicate that measured and simulated reflection coefficients are matched in both cases of HSA and conventional types. These antennas resonate at $2.62 \mathrm{GHz}$ and suppress the third harmonic frequency at $7.86 \mathrm{GHz}$. The measured bandwidths of the proposed RDGS and CDGS antennas at the center frequency of $2.62 \mathrm{GHz}$ are about $250 \mathrm{MHz}(9.5 \%)$ and $400 \mathrm{MHz}$ (15.2\%), respectively. The difference between simulation and measured bandwidths at the fundamental frequency in Figure 9(a) is due to power leakage from the used SMA connector and to the loss of energy to the surface waves.

As seen from Figures 8(a) and 9(a), the reflection coefficient between 4 and $10 \mathrm{GHz}$, especially near $7.8 \mathrm{GHz}$ is about zero with a maximum ripple of $2.4 \mathrm{~dB}$. Thus, the third harmonic is well suppressed up to $23 \mathrm{~dB}$ in RDGS and $22 \mathrm{~dB}$ in CDGS. In addition, it is observed from Figure 8(b) that the first mode $(53 \Omega+j 5 \Omega)$ of the RDGS antenna is matched while the second $(2 \Omega+j 8 \Omega)$ and third $(1 \Omega-j 47 \Omega)$ modes are suitably suppressed. Similarly, as shown in Figure 9(b), the first mode $(52 \Omega-j 8 \Omega)$ of the CDGS antenna is also matched while the second $(1 \Omega-j 22 \Omega)$ and third $(3.5 \Omega+$ $j 27 \Omega$ ) modes are suppressed, acceptably.

According to the results, the proposed slot antennas having a simple structure and low design complexity are suitable choices for harmonic degradation. The photographs of the two realized RDGS and CDGS wide slot antennas are illustrated in Figure 10. As an example, the constructed prototype of RDGS slot antenna was measured in an 


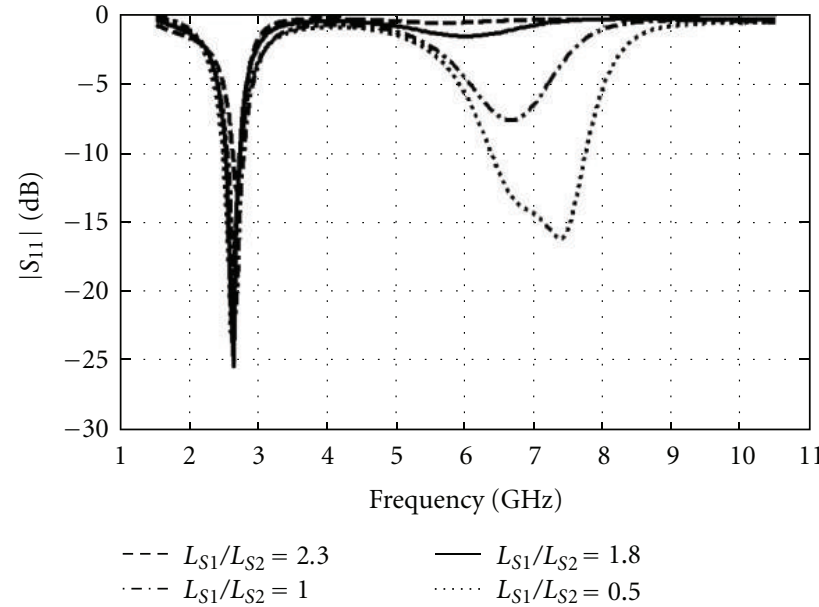

(a)

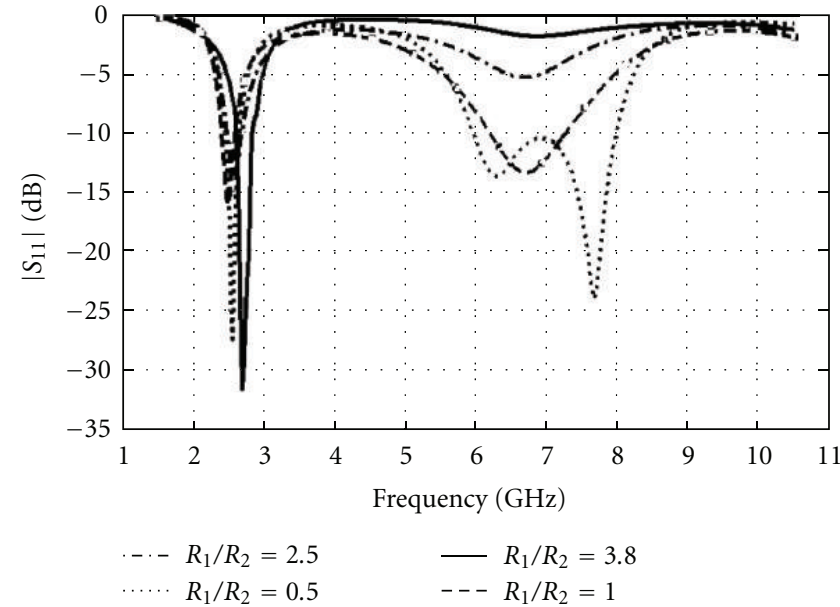

(b)

FIGURE 7: Simulated reflection coefficients of (a) RDGS slot antenna versus ratios of $L_{S 1} / L_{S 2}$ and (b) CDGS slot antenna versus ratios of $R_{1} / R_{2}$. The other parameters are constant and seen in Figure 1 .

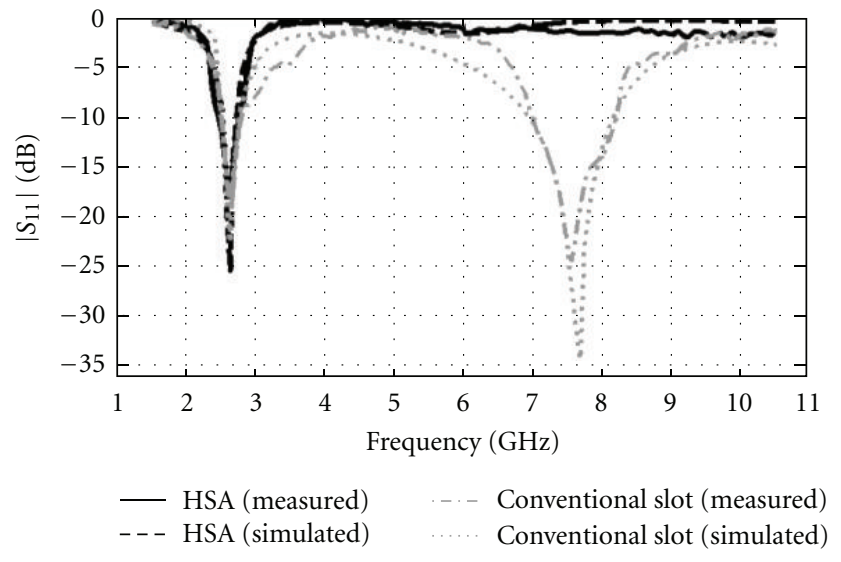

(a)

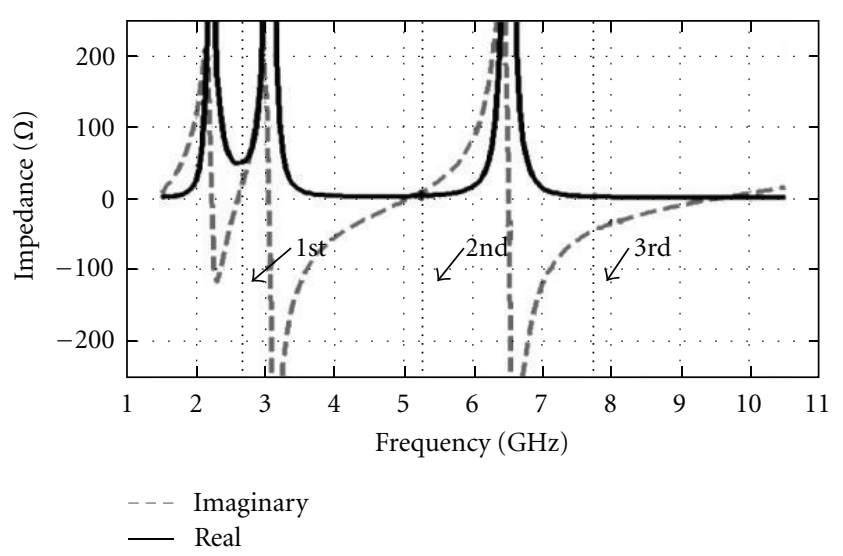

(b)

FIGURE 8: (a) Reflection coefficients of the RDGS slot antenna and (b) measured input impedance of the RDGS slot antenna.

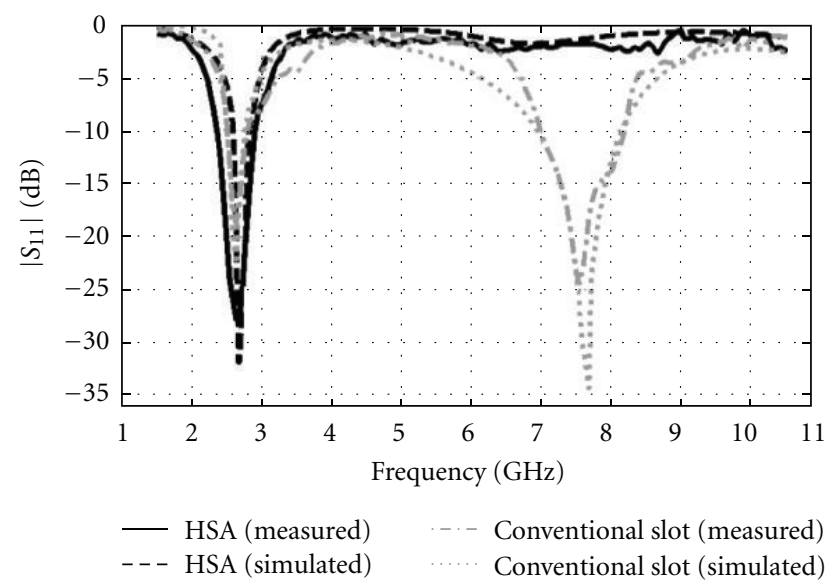

(a)

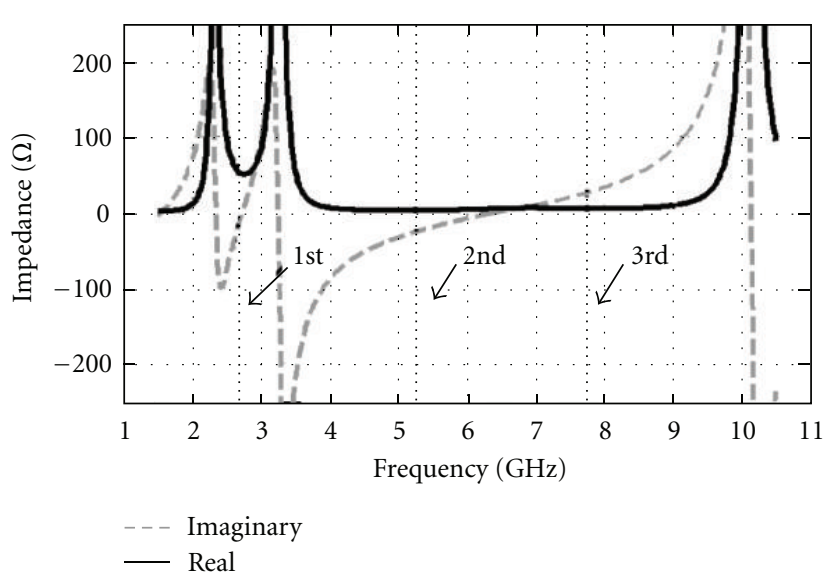

(b)

FIGURE 9: (a) Reflection coefficients of the CDGS slot antenna and (b) measured input impedance of the CDGS slot antenna. 


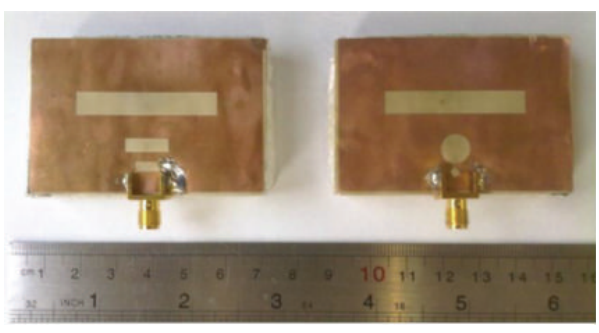

(a)

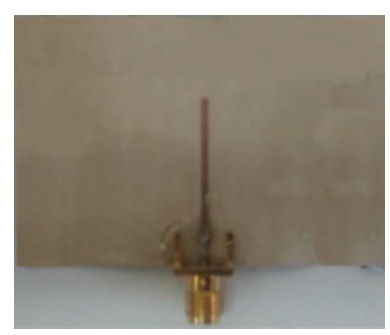

(b)

FIGURE 10: Photographs of the fabricated RDGS and CDGS wide slot antennas: (a) back and (b) front.

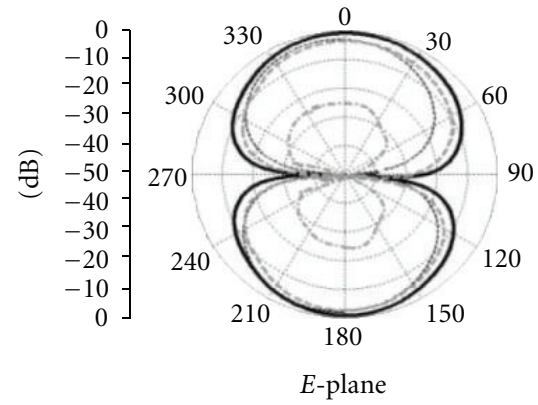

Conventional slot, $2.62 \mathrm{GHz}$

- Conventional slot, $7.86 \mathrm{GHz}$

- - HSA, $2.62 \mathrm{GHz}$

HSA, $7.86 \mathrm{GHz}$

(a)

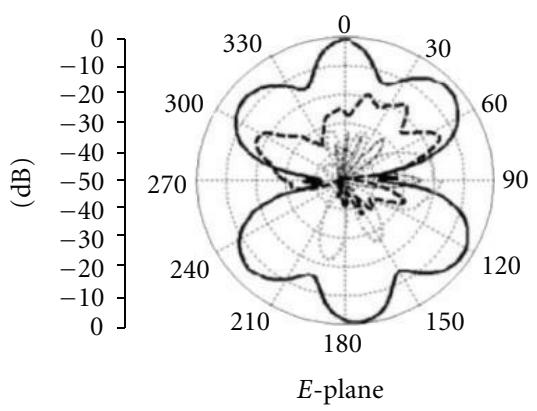

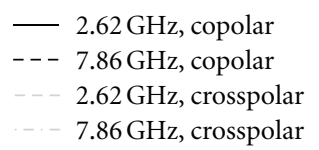

(c)

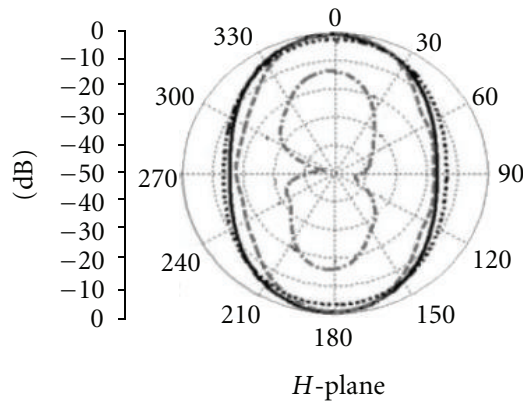

Conventional slot, $2.62 \mathrm{GHz}$

- Conventional slot, $7.86 \mathrm{GHz}$

- - - HSA, $2.62 \mathrm{GHz}$

HSA, $7.86 \mathrm{GHz}$

(b)
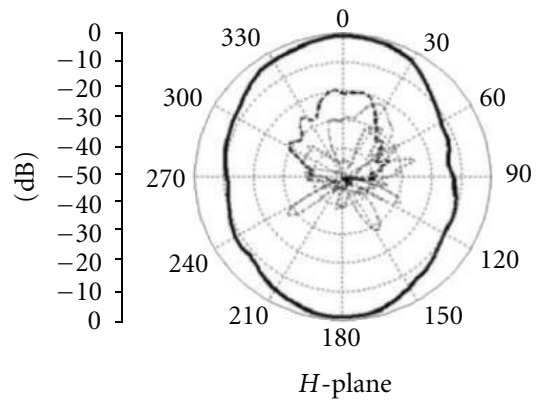

- $2.62 \mathrm{GHz}$, copolar

- - $7.86 \mathrm{GHz}$, copolar $2.62 \mathrm{GHz}$, crosspolar $7.86 \mathrm{GHz}$, crosspolar

(d)

FIGURE 11: Simulated radiation patterns of both RDGS slot antenna and conventional slot antenna: (a) E-plane and (b) H-plane as well as measured patterns of only RDGS antenna: (c) E-plane and (d) H-plane.

anechoic chamber for studying and comparison of the radiation characteristics.

Figure 11 shows the measured and simulated radiation patterns of the both RDGS and conventional slot antennas at the fundamental and third harmonic frequencies. The patterns are omnidirectional in the $\mathrm{H}$-plane and bidirectional in the E-plane as in a center-fed slot antenna. As seen in Figures 11(a) and 11(c), for the normalized peak power of the fundamental frequency, the simulated and measured Eplane radiation patterns of the proposed RDGS slot antenna at the third harmonic of $7.86 \mathrm{GHz}$ in the broadside direction are less than -25 and $-27 \mathrm{~dB}$, respectively. Similarly, Figures $11(\mathrm{~b})$ and 11(d) also show that the simulated and measured $\mathrm{H}$-plane patterns of the RDGS slot antenna at the third harmonic frequency are less than -17 and $-20 \mathrm{~dB}$, respectively. These results show that the radiation patterns at the harmonic frequency are acceptably suppressed. Moreover, the measured maximum gains of the proposed RDGS slot antenna at 2.62 and $7.86 \mathrm{GHz}$ are 2.8 and $0.8 \mathrm{dBi}$, respectively, while the same values for the conventional 
antenna are 3 and $7.9 \mathrm{dBi}$, respectively. It should be noticed that the $3 \mathrm{D}$ radiation patterns at the fundamental frequency in conventional and harmonic suppression antennas are nearly similar to each other with a stable shape which means two proposed filtering slots have not been destructed by the performance of the main radiating slot at $2.62 \mathrm{GHz}$.

\section{Conclusion}

In this paper, two microstrip slot antennas using rectangular and circular defected ground structures (DGSs) with harmonic suppression characteristic over harmonic frequency band have been proposed and investigated. The reflection coefficients were less than $-2.4 \mathrm{~dB}$ at the second and third harmonic frequencies compared to those of the conventional slot antenna. The measured and simulated radiation patterns in the HSAs indicate at least $17 \mathrm{~dB}$ suppression at the third harmonic frequency. According to the measured results, these antennas with the simple harmonic suppression structures are quite effective for harmonic suppression. Therefore, the proposed antennas can be suitable for active integrated antennas.

\section{Acknowledgment}

The authors thank the Iran's Telecommunication Educational Research Center (ITERC) for its financial support.

\section{References}

[1] V. Radisic, Y. Qian, and T. Itoh, "Novel architectures for high-efficiency amplifiers for wireless applications," IEEE Transactions on Microwave Theory and Techniques, vol. 46, no. 11, pp. 1901-1909, 1998.

[2] V. Radisic, Y. Qian, and T. Itoh, "Broadband power amplifier integrated with slot antenna and novel harmonic tuning structure," in Proceedings of the IEEE MTT-S International Microwave Symposium, vol. 3, pp. 1895-1898, June 1998.

[3] Y. Horii and M. Tsutsumi, "Harmonic control by photonic bandgap on microstrip patch antenna," IEEE Microwave and Wireless Components Letters, vol. 9, no. 1, pp. 13-15, 1999.

[4] Y. J. Sung, M. Kim, and Y. S. Kim, "Harmonics reduction with defected ground structure for a microstrip patch antenna," IEEE Antennas and Wireless Propagation Letters, vol. 2, pp. 111-113, 2003.

[5] Y. J. Sung and Y. S. Kim, "An improved design of microstrip patch antennas using photonic bandgap structure," IEEE Transactions on Antennas and Propagation, vol. 53, no. 5, pp. 1799-1804, 2005.

[6] N. A. Nguyen, R. Ahmad, Y. T. Im, Y. S. Shin, and S. O. Park, "A T-shaped wide-slot harmonic suppression antenna," IEEE Antennas and Wireless Propagation Letters, vol. 6, pp. 647-650, 2007.

[7] H. Kim and Y. J. Yoon, "Microstrip-fed slot antennas with suppressed harmonics," IEEE Transactions on Antennas and Propagation, vol. 53, no. 9, pp. 2809-2817, 2005.

[8] M. S. Ghaffarian and G. Moradi, "A novel harmonic suppressed coplanar waveguide (CPW)-fed slot antenna," IEEE Antennas and Wireless Propagation Letters, vol. 10, Article ID 5959181, pp. 788-791, 2011.
[9] N. C. Karmakar, S. M. Roy, and I. Balbin, "Quasi-static modeling of defected ground structure," IEEE Transactions on Microwave Theory and Techniques, vol. 54, no. 5, pp. 21602168, 2006.

[10] M. S. Joung, J. S. Park, and H. S. Kim, "A novel modeling method for defected ground structure using adaptive frequency sampling and its application to microwave oscillator design," IEEE Transactions on Magnetics, vol. 41, no. 5, pp. 1656-1659, 2005.

[11] H. G. Akhavan and D. Mirshekar-Syahkal, "Approximate model for microstrip fed slot antennas," Electronics Letters, vol. 30, no. 23, pp. 1902-1903, 1994. 

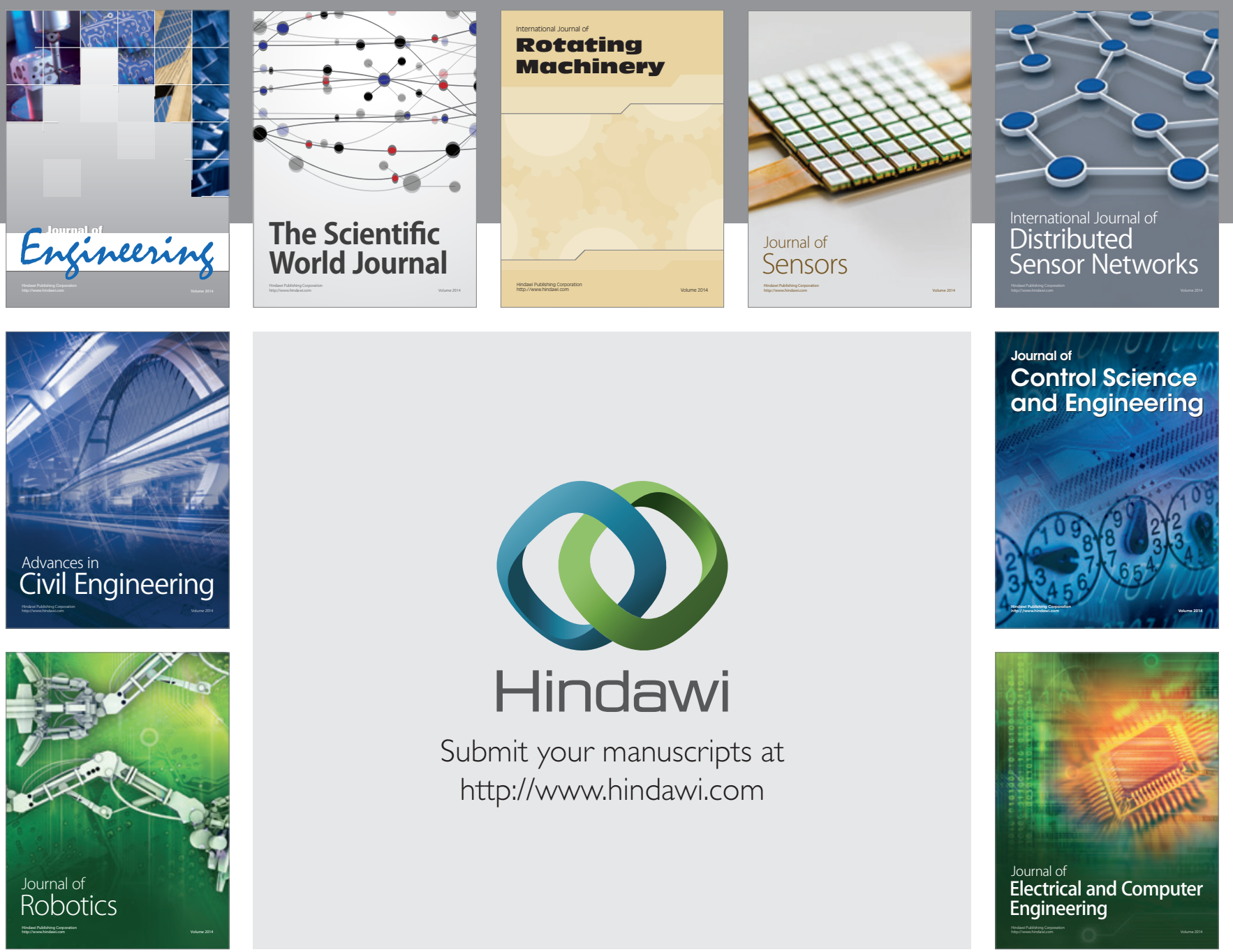

Submit your manuscripts at

http://www.hindawi.com
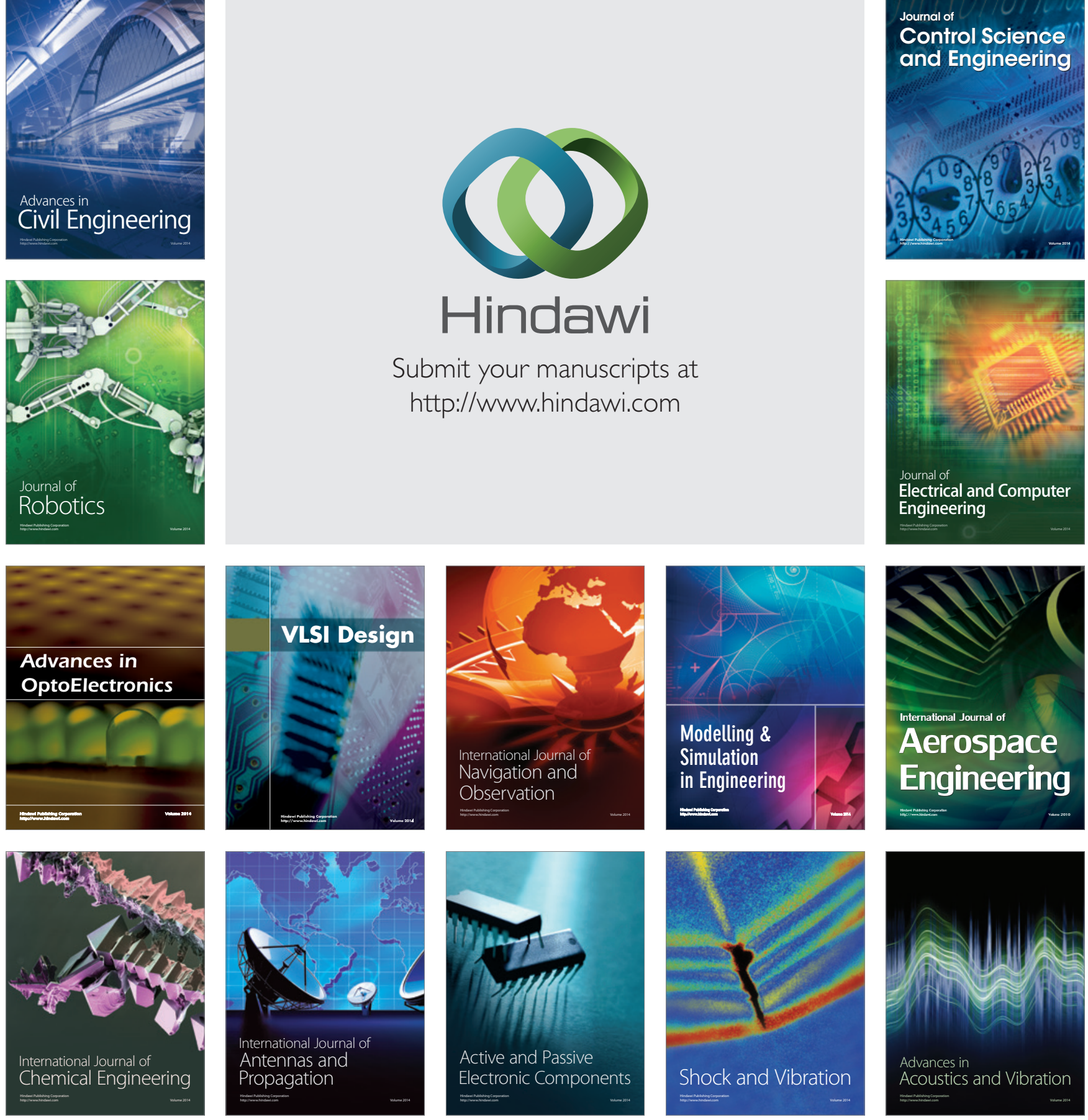